2003

\title{
Synchronization Of Spatiotemporal Patterns In Locally Coupled Excitable Media
}

Michael Hildebrand

Jianxia Cui

Eugene Mihaliuk

Jichang Wang

Kenneth Showalter

Follow this and additional works at: https://researchrepository.wvu.edu/faculty_publications

\section{Digital Commons Citation}

Hildebrand, Michael; Cui, Jianxia; Mihaliuk, Eugene; Wang, Jichang; and Showalter, Kenneth, "Synchronization Of Spatiotemporal Patterns In Locally Coupled Excitable Media" (2003). Faculty Scholarship. 692.

https://researchrepository.wvu.edu/faculty_publications/692 


\title{
Synchronization of spatiotemporal patterns in locally coupled excitable media
}

\author{
Michael Hildebrand, ${ }^{1,2}$ Jianxia Cui, ${ }^{1}$ Eugene Mihaliuk, ${ }^{1}$ Jichang Wang, ${ }^{1,3}$ and Kenneth Showalter ${ }^{1,2}$ \\ ${ }^{1}$ Department of Chemistry, West Virginia University, Morgantown, West Virginia 26506-6045, USA \\ ${ }^{2}$ Fritz-Haber-Institut der Max-Planck-Gesellschaft, Faradayweg 4-6, D-14195 Berlin, Germany \\ ${ }^{3}$ Department of Chemistry and Biochemistry, University of Windsor, Windsor, Ontario, Canada N9B 3P4
}

(Received 20 March 2003; published 14 August 2003)

\begin{abstract}
The synchronization of two distributed Belousov-Zhabotinsky systems is experimentally and theoretically investigated. Symmetric local coupling of the systems is made possible with the use of a video cameraprojector scheme. The spatial disorder of the coupled systems, with random initial configurations of spirals, gradually decreases until a final state is attained, which corresponds to a synchronized state with a single spiral in each system. The experimental observations are confirmed with numerical simulations of two identical Oregonator models with symmetric local coupling, and a systematic study reveals generalized synchronization of spiral waves. Several different types of synchronization attractors are distinguished.
\end{abstract}

DOI: 10.1103/PhysRevE.68.026205

PACS number(s): 82.40.Qt, 05.45.Xt, 05.65.+b, 47.54.+r

Synchronization phenomena are of fundamental importance in physical, chemical, biological, and technical systems. The synchronization of coupled chaotic oscillators has attracted much attention in recent years, and complete, phase, lag, and generalized synchronization have been distinguished in such systems [1]. These concepts have been applied in the analysis and interpretation of experimental data from the cardiorespiratory system [2], paddlefish cells [3], the human brain [4], and in the contexts of population dynamics [5] and communication with chaotic lasers [6]. Recently, synchronization phenomena in spatially extended systems have attracted increasing attention. Identical synchronization and phase synchronization have been observed in systems exhibiting spatiotemporal chaos [7-9].

In this paper, we describe experimental and theoretical studies of the synchronization of two locally coupled domains of excitable media exhibiting spiral wave behavior. We have used the photosensitive Belousov-Zhabotinsky (BZ) reaction [10], which is particularly convenient for studies on influencing existing patterns or generating new ones by the application of various types of external forcing [11-13], or by local [14], nonlocal [15], or global [16] feedback mechanisms. The identical synchronization of chemical wave patterns has been previously observed in a BZ system with diffusive cross-membrane coupling [17]. Here we study two domains of excitable media that are locally coupled to each other by means of a video camera-video projector setup through a coupling algorithm, which leads to weaker than identical synchronization [18].

Prior to each experiment, the projected image was adjusted at each pixel by an iterative algorithm to ensure a spatially uniform illumination field, upon which all subsequent projected images were based. The local concentration of oxidized catalyst was recorded with a video camera, and the recorded image was divided into an array of square cells. In all experiments, the lateral size of each cell was much smaller than the spiral wavelength. The medium was partitioned into two square regions separated and surrounded by an unexcitable boundary generated with high-intensity light. The corresponding cells in the two regions were locally coupled by an illumination field projected onto the gel me- dium through a $460 \mathrm{~nm}$ bandpass filter. Bromide ions are locally produced in a photochemical cycle, and, as a result, the local excitability is appropriately modified [20].

The intensity $I_{i}(\mathbf{r}, t)(i=1,2)$ of the projected illumination field was computed as

$$
I_{i}(\mathbf{r}, t)=I_{0} \Theta_{i} H\left(\Theta_{i}\right),
$$

where $I_{0}=7.84 \mathrm{~mW} / \mathrm{cm}^{2}$ is the background light intensity, $H(z)$ is the step function [with $H(z)=1$ for $z \geqslant 0$ and $H(z)=0$ for $z<0]$, and the functions $\Theta_{i}$ are defined as

$$
\Theta_{1,2}(\mathbf{r}, t)=1 \pm \kappa\left[v_{1}(\mathbf{r}, t)-v_{2}(\mathbf{r}, t)\right],
$$

where the plus and the minus signs refer to systems 1 and 2 , respectively, $v_{i}(\mathbf{r}, t)$ is the local concentration of the oxidized catalyst in the $i$ th system [21], and $\kappa$ specifies the strength of the symmetric mutual coupling.

Experiments were carried out with initial conditions consisting of a random configuration of spirals [Fig. 1(a)], generated by an algorithm for light-mediated spatiotemporal noise [22]. In the absence of coupling, the number of spirals in both systems did not change over the course of the experiment. The number continuously decreased, however, when the corresponding elements in the two domains were locally coupled according to Eq. (1). In the first stage of the evolution, spirals were removed from each zone by collision with counter-rotating spirals in the same zone or with the boundaries of the system [Fig. 1(b)] until only a few spirals remained [Fig. 1(c)]. The coupled system eventually relaxed to one of three possible final states. The most common outcome was a single spiral in each zone, with both spirals rotating in the same direction [Fig. 1(d)]. Two other final states were occasionally observed, with one zone containing a single spiral and the other in the uniform steady state or with both zones in the uniform steady state.

The experimental results shown in Figs. 1(a)-(d) can be qualitatively reproduced by numerical simulations of two locally coupled Oregonator models [23], Figs. 1(e)-(h). The model includes the additional terms $\phi_{i}(\mathbf{r}, t)(i=1,2)$, taking into account the photochemical bromide production $[20,24]$ : 


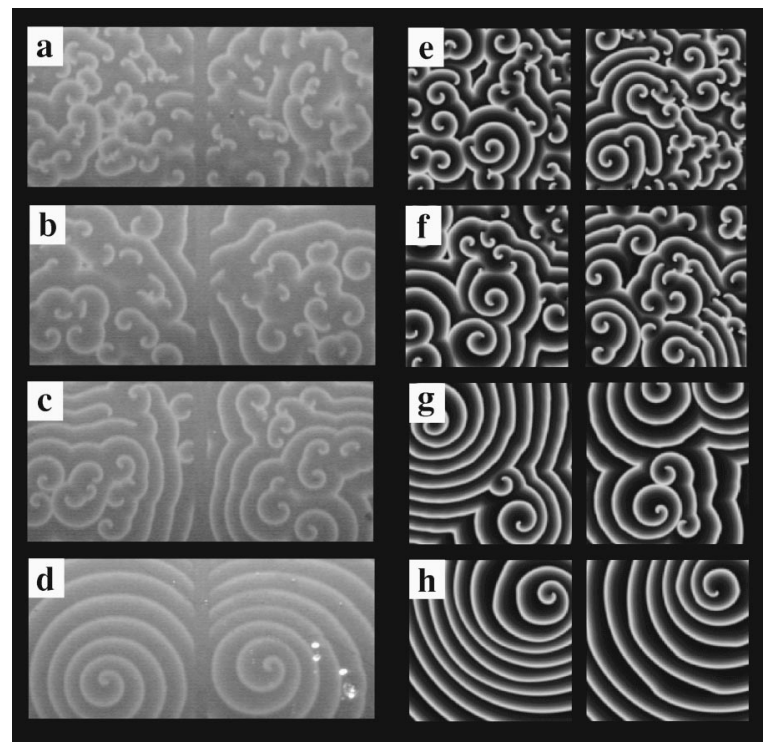

FIG. 1. Relaxation of an initially disordered array of spirals to a synchronized state in two locally coupled excitable media. (a)-(d) Experimental behavior of locally coupled BZ systems with $I_{0}$ $=7.84 \mathrm{~mW} / \mathrm{cm}^{2}, T=6.5^{\circ} \mathrm{C}$, and $\kappa=0.31$. Left and right panels of each snapshot show the distribution of $\mathrm{Ru}(\mathrm{bpy}) 3_{3}^{3+}$ (in gray scale increasing from black to white) at $t=2.30 \mathrm{~min}$ (a), $12.25 \mathrm{~min}$ (b), $30.00 \mathrm{~min}$ (c), and $76.52 \mathrm{~min}$ (d) after starting the experiment. The total illumination field $\left(7.59 \times 3.51 \mathrm{~cm}^{2}\right)$ was divided into an array of $362 \times 167$ square cells and updated every $2.0 \mathrm{~s}$. (e) $-(\mathrm{h})$ Numerical simulations of Eq. (3) for $\phi_{0}=0.035, \kappa=1, \varepsilon=0.03, f=1$, and $q=0.0015$ in a system of size $L=100$. Left and right panels of each snapshot show $v_{1}(x, y, t)$ and $v_{2}(x, y, t)$ (in gray scale increasing from black to white) at $t=91.9$ (f), 612.5 (g), and 918.7 (h) after starting the simulation with the distribution shown in (e).

$$
\begin{gathered}
\frac{\partial u_{1}}{\partial t}=\nabla^{2} u_{1}+\frac{1}{\varepsilon}\left\{u_{1}-u_{1}^{2}-\left[f v_{1}+\phi_{1}(\mathbf{r}, t)\right] \frac{u_{1}-q}{u_{1}+q}\right\}, \\
\frac{\partial v_{1}}{\partial t}=u_{1}-v_{1}, \\
\frac{\partial u_{2}}{\partial t}=\nabla^{2} u_{2}+\frac{1}{\varepsilon}\left\{\begin{array}{c}
\left.u_{2}-u_{2}^{2}-\left[f v_{2}+\phi_{2}(\mathbf{r}, t)\right] \frac{u_{2}-q}{u_{2}+q}\right\}, \\
\frac{\partial v_{2}}{\partial t}=u_{2}-v_{2},
\end{array}\right.
\end{gathered}
$$

where the variables $u_{i}$ and $v_{i}$ correspond to the dimensionless concentrations in the $i$ th system of the autocatalytic species $\mathrm{HBrO}_{2}$ and the oxidized catalyst, respectively, $\varepsilon$ and $q$ are scaling parameters, and $f$ is an adjustable stoichiometry parameter. The rates of bromide production from irradiation $\phi_{i}(\mathbf{r}, t)$ are proportional to the respective light intensities $I_{i}(\mathbf{r}, t)$, as given by Eq. (1) (with the proportionality factor $\left.\phi_{0} / I_{0}\right)$.

Insights into the complex relaxation behavior shown in Fig. 1 can be gleaned from systematic numerical simulations with a single spiral in each system. The synchronization behavior depends on the kinetic parameters, the coupling
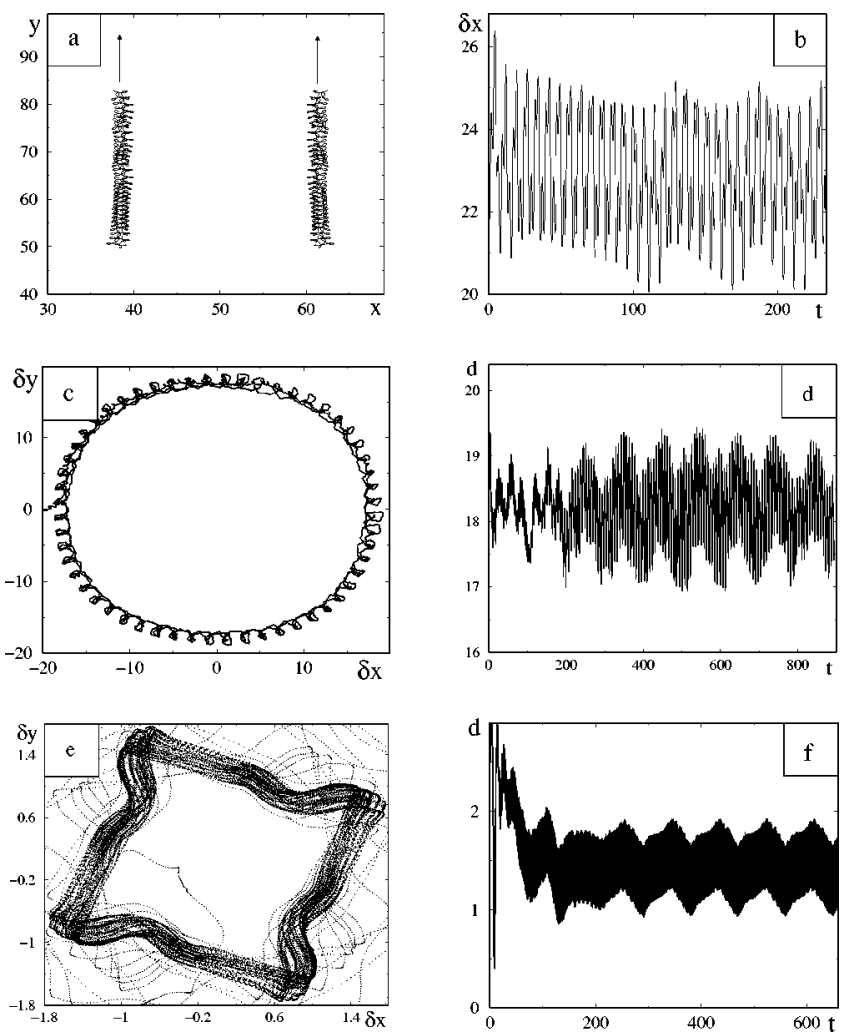

FIG. 2. Results from numerical simulations of Eq. (3) for a single spiral in each system. (a) Trajectories of spiral tips in the coupled systems 1 (left) and 2 (right), where the spirals rotate in opposite directions; $d_{i}=20, t=235.6$. (b) Time series of the horizontal distance $\delta x$ between the spiral tips shown in (a). (c) Coupled spirals rotating in the same direction. Trajectory of the difference vector between the spiral tips in systems 1 and 2 for $d_{i}=20, t$ $=895.1$. (d) Time series corresponding to the distance $d(t)$ between the two spiral tips in (c). (e) Coupled spirals rotating in the same direction. Trajectory of the difference vector between the spiral tips in systems 1 and 2 for $d_{i}=2.5, t=706.8$. (f) Time series corresponding to the distance $d(t)$ between the two spiral tips in (e). All other parameters as in Figs. 1(e)-(h).

strength, the relative sense of rotation of the two spirals, and their initial separation distance $d_{i}$ [25]. When the two spirals rotate in opposite directions, the coupling leads to a parallel drift of the spiral tips until they annihilate by collision with the no-flux boundary of the system [see Figs. 2(a) and 2(b)]. This behavior was observed for values of the coupling strength $\kappa$ below 2.9 and as low as $\kappa=5 \times 10^{-3}$ [with the parameter values as in Figs. 1(e)-(h)]. For stronger coupling, the spirals were destroyed by breakup, and both systems relaxed to the uniform stationary state.

A completely different scenario is observed when the two spirals are corotating. For intermediate values of the coupling strength $(2.9>\kappa>0.03)$, complex synchronization behavior is observed, as shown in Figs. 2(c)-(f). If the initial distance between the spiral tips is chosen sufficiently large $\left(d_{i}>d_{i}^{c}\right)$, the tips drift on opposite sides of an approximately circular trajectory, leading to a nearly circular trajectory for the difference vector of the two tip positions, shown in Fig. 2(c). The modulations visible in the trajectory result from the 

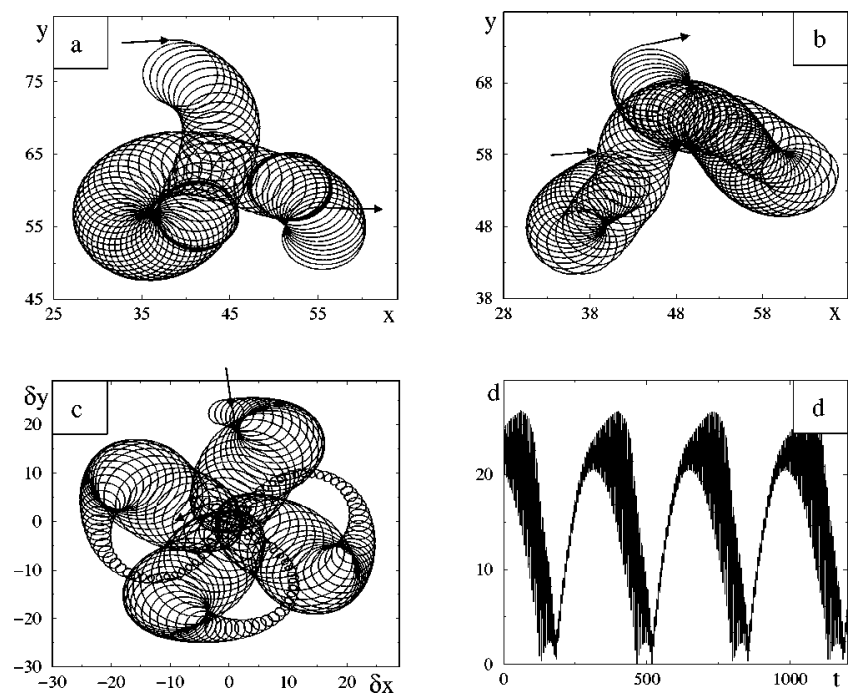

FIG. 3. Results from a numerical simulation of Eq. (3) with clockwise rotating spirals; $\phi_{0}=0.01, \kappa=1, \varepsilon=0.05, f=3.5, q$ $=0.002, L=120$, and the initial spiral distance $d_{i}=7$. (a,b) Trajectories of spiral tips in the coupled systems 1 (a) and 2(b) for $t$ $=602$. The arrows mark the beginning and end points of the trajectories. (c) Trajectory of the difference vector between the two tip positions. (d) Time series of the distance $d(t)$ between the two spiral tips; $t=1203.8$ in (c) and (d).

underlying meandering motion of the tips. A perfect circle is obtained when the spiral tips exhibit steady rotation in the uncoupled system. Upon varying the initial distance $d_{i}$, we find a discrete set of such attractors. For example, for the parameters chosen in Fig. $2, d_{i}^{c} \approx 12$, and only the (average) diameters $d_{\infty}=32.5,72.5,112.5,155, \ldots$ of the approximately circular trajectory are exhibited for $t \rightarrow \infty$, depending on the initial distance $d_{i}$. Similar trajectories of a spiral tip have been observed in feedback-controlled dynamics of meandering spirals, and have been coined "resonance attractors" [14]. A theory explaining the existence and the stability of these attractors has been developed [26], and a similar approach can be used to understand the nature of attractors like that in Fig. 2(c) [27].

For $d_{i}<d_{i}^{c}$, a different type of synchronized tip motion occurs. The spiral tips now perform a meandering motion around a common central point. The trajectory of the difference vector of the two tip positions is more localized and clearly deviates from a circular shape, as shown in Fig. 2(e). This behavior resembles the "entrainment attractors" observed in feedback-controlled dynamics of meandering spirals [14]. For both large and small $d_{i}$, the spirals are destroyed by breakup for $\kappa>2.9$, and both systems relax to the uniform stationary state.

For other values of the system parameters, e.g., when the spirals perform steady rotation in the uncoupled system, a different kind of synchronization can be observed. Figures 3(a) and 3(b) show tip trajectories with an irregular drift superimposed on the steady spiral tip rotation. A careful examination of the two trajectories shows that, at certain moments, the tip in system 1 stops its drift motion and exhibits a localized rotation for a brief period until the tip in system 2 closely approaches, whereupon the two spiral tips again begin to separate. The continuous repetition of this behavior leads to the formation of a complex flower pattern for the difference vector between the two tip positions, which is centered around $(0,0)$ [Fig. 3(c)]. The weak synchronization of the two spirals is apparent in the time series of the tip distance shown in Fig. 3(d). Quasi-mixed-mode oscillations are observed, with the period of the large-scale oscillation corresponding to the time needed for completing one of the petals shown in Fig. 3(c), and the period of the small-scale oscillation being determined by the underlying rotational period of a spiral in the uncoupled system.

Note that in all of the cases shown in Figs. 2 and 3, the coupling induces a drift of the spiral tips, which is superimposed on the localized motion observed in the absence of coupling. Thus, for a given spiral in one of the irregular arrays shown in Fig. 1, the coupling leads to an increase in the translational motion of this spiral. Hence, the probability that its tip will meet another tip with opposite charge, or that it will collide with the system boundary and thus be annihilated, is increased. The total number of spirals therefore decreases more rapidly than in the absence of coupling, where an initially disordered array of spirals is extremely long lived as a result of the exponentially weak interactions of the screened spiral tips [28]. We have also investigated the generality of the synchronization behavior by carrying out numerical simulations for a system of coupled Barkley models [29] displaying spiral behavior. Upon increasing the coupling strength, we observe a transition from localized tip motion to the stronger synchronization displayed in Fig. 2(c) via the synchronization shown in Fig. 3.

The synchronization found in this study is related to that found in a study of cross-membrane coupling of spatiotemporal behavior in the BZ reaction [17]; however, there are significant differences between the two systems and their synchronization behavior. Identical synchronization was found in the cross-membrane study, where waves on each side of a semipermeable membrane interact via activating messenger species, such as $\mathrm{HBrO}_{2}$ and $\mathrm{BrO}_{2}$, which are able to pass through the membrane interior. The symmetrical coupling studied here arises from the inhibiting effects of 460-nm light, which interacts with $\mathrm{Ru}(\mathrm{bpy})_{3}^{2+}$ to give rise to a photochemical process that generates $\mathrm{Br}^{-}$, an inhibitor of autocatalysis [20]. The synchronization is weaker than identical synchronization, as the remaining single spiral waves in each system differ in position and phase, as shown in Fig. 1. Our numerical studies have also revealed a form of generalized synchronization, with a variety of synchronization attractors akin to the resonance attractors described in Ref. [14].

In summary, we have shown that the local coupling of two excitable media with complex spiral patterns gives rise to a reduction in spatial disorder, ultimately leading to a coherent pattern in each system corresponding to either a single spiral wave or to the uniform stationary state. Numerical simulations with a single spiral in each system show that instead of identical synchronization, different types of generalized synchronization attractors are exhibited. To our knowledge, this 
constitutes the first example of generalized synchronization in coupled spatially extended systems with nonchaotic dynamics. We note that the coupling is multiplicative and symmetric, which would be anticipated in complex biological systems.
M.H. and K.S. are grateful for support by the Alexander von Humboldt-Stiftung. K.S. thanks the National Science Foundation (Grant No. CHE-9974336) and the Office of Naval Research (Grant No. N00014-01-1-0596) for supporting this research.
[1] A. Pikovsky, M. Rosenblum, and J. Kurths, Synchronization-A Universal Concept in Nonlinear Sciences (Cambridge University Press, Cambridge, 2001); S. Boccaletti, J. Kurths, G. Osipov, D.L. Valladares, and C.S. Zhou, Phys. Rep. 366, 1 (2002).

[2] C. Schäfer, M.G. Rosenblum, J. Kurths, and H.-H. Abel, Nature (London) 392, 239 (1998).

[3] A. Neiman, X. Pei, D. Russell, W. Wojtenek, L. Wilkens, F. Moss, H.A. Braun, M.T. Huber, and K. Voigt, Phys. Rev. Lett. 82, 660 (1999).

[4] P. Tass, M.G. Rosenblum, J. Weule, J. Kurths, A. Pikovsky, J. Volkmann, A. Schnitzler, and H.J. Freund, Phys. Rev. Lett. 81, 3291 (1998).

[5] B. Blasius, A. Huppert, and L. Stone, Nature (London) 399, 354 (1999).

[6] G.D. VanWiggeren and R. Roy, Science 279, 1198 (1998).

[7] P. Parmananda, Phys. Rev. E 56, 1595 (1997).

[8] J. Bragard, S. Boccaletti, and F.T. Arecchi, Int. J. Bifurcation Chaos Appl. Sci. Eng. 11, 2715 (2001).

[9] L. Junge and U. Parlitz, Phys. Rev. E 62, 438 (2000).

[10] L. Kuhnert, K.I. Agladze, and V. Krinsky, Nature (London) 337, 244 (1989).

[11] O. Steinbock, V.S. Zykov, and S.C. Müller, Nature (London) 366, 322 (1993).

[12] V. Petrov, Q. Ouyang, and H.L. Swinney, Nature (London) 388, 655 (1997); A.L. Lin, A. Hagberg, A. Ardelea, M. Bertram, H.L. Swinney, and E. Meron, Phys. Rev. E 62, 3790 (2000).

[13] I. Sendiña-Nadal, E. Mihaliuk, J. Wang, V. Pérez-Muñuzuri, and K. Showalter, Phys. Rev. Lett. 86, 1646 (2001).

[14] S. Grill, V.S. Zykov, and S.C. Müller, Phys. Rev. Lett. 75, 3368 (1995).

[15] M. Hildebrand, H. Skødt, and K. Showalter, Phys. Rev. Lett. 87, 088303 (2001).

[16] V.K. Vanag, L. Yang, M. Dolnik, A.M. Zhabotinsky, and I.R. Epstein, Nature (London) 406, 389 (2000).

[17] D. Winston, M. Arora, J. Maselko, V. Gáspár, and K. Showalter, Nature (London) 351, 132 (1991).

[18] Experiments were carried out with the catalyst of the lightsensitive $\mathrm{BZ}$ reaction, $\mathrm{Ru}(\mathrm{bpy})_{3}^{2+}$, immobilized in a thin slab of silica gel. The silica gel medium was prepared by acidifying a solution of $10 \%(w / w) \mathrm{Na}_{2} \mathrm{SiO}_{3}$ and $2.0 \mathrm{mM} \mathrm{Ru}(\mathrm{bpy})_{3}^{2+}$ (where bpy $=2,2^{\prime}$-bipyridyl) with $\mathrm{H}_{2} \mathrm{SO}_{4}$ and casting a uniform $0.3 \times 75.9 \times 35.1 \mathrm{~mm}^{3}$ layer onto a microscopic slide. The gel was continuously fed with fresh, catalyst-free BZ solution in a thermostated reactor to maintain constant, nonequilibrium conditions [19]. The chemical composition of the reaction mixture was $0.28 \mathrm{MBrO}_{3}^{-}, 0.05 \mathrm{M}$ malonic acid, $0.165 \mathrm{M}$ bromomalonic acid, and $0.4 \mathrm{MH}_{2} \mathrm{SO}_{4}$, corresponding to excitable conditions where meandering spirals are observed in the unperturbed system.

[19] Z. Noszticzius, W. Horsthemke, W.D. McCormick, H.L. Swinney, and W.Y. Tam, Nature (London) 329, 619 (1987).

[20] S. Kádár, T. Amemiya, and K. Showalter, J. Phys. Chem. A 101, 8200 (1997).

[21] The gray level is linearly proportional to concentration over the experimental range, where the camera response to intensity is logarithmic. We measure gray level between 0 and 255 and divide by 255 to obtain the scaled concentration, which we treat as dimensionless.

[22] J. Wang, S. Kádár, P. Jung, and K. Showalter, Phys. Rev. Lett. 82, 855 (1999).

[23] R.J. Field and R.M. Noyes, J. Chem. Phys. 60, 1877 (1974); J.J. Tyson and P.C. Fife, ibid. 73, 2224 (1980).

[24] H.J. Krug, L. Pohlmann, and L. Kuhnert, J. Phys. Chem. 94, 4862 (1990).

[25] The separation distance is the distance between the spiral tips in the composite image composed of the pixelwise superposition of the two systems. The superposition is exactly the same as that during the coupling of the systems.

[26] A. Karma and V.S. Zykov, Phys. Rev. Lett. 83, 2453 (1999); V.S. Zykov, O.U. Kheowan, O. Rangsiman, and S.C. Müller, Phys. Rev. E 65, 026206 (2002).

[27] J. Cui, M. Hildebrand, and K. Showalter (unpublished).

[28] I.S. Aranson, L. Kramer, and A. Weber, Physica D 53, 376 (1991).

[29] D. Barkley, Physica D 49, 61 (1991). The coupling was introduced by renormalizing the parameter $b$ for the excitability threshold as in Eq. (2). 